\title{
NORMS OF INTEGRABLE CUSP FORMS
}

\author{
L. ALAYNE PARSON
}

(Communicated by Larry J. Goldstein)

\begin{abstract}
The norms of modular cusp forms, viewed as belonging to the Bers' spaces of integrable and bounded forms, are estimated in terms of the Fourier coefficients of the cusp form.
\end{abstract}

In [5] R. A. Smith found upper and lower bounds for the $L^{2}$-norms of Maass wave functions which were also simultaneous eigenfunctions of the Hecke operators. His bounds depended on the size of the first Fourier coefficient $c_{f}(1)$ of $f$ and elucidated the connection between $\left|c_{f}(1)\right|$ and the $L^{2}$-norm of $f$ under two different normalizations of $f$. Analogous results are examined here for cusp forms on subgroups of the classical modular group which can be viewed as belonging to the Bers' spaces $B_{r}(G), A_{r}(G)$, and $A_{r}^{2}(G)$. Estimates are given which have bearing on the numerical calculation of the various norms and which emphasize the connection between the first nonzero Fourier coefficient of the cusp form and the value of the norm.

1. Definitions and notation. Let $G$ denote a subgroup of finite index in the classical modular group $G(1)$. A function $f$ analytic in the upper half-plane $\mathscr{H}$ is a cusp form on $G$ of positive weight $r$ with multiplier system $\nu$ of modulus one if for all $z \in \mathscr{H}$

$$
f(M z)=\nu(M)(c z+d)^{r} f(z) \text { for each } M=\left(\begin{array}{ll}
a & b \\
c & d
\end{array}\right) \in G
$$

and $f(z) \rightarrow 0$ as $z$ approaches any cusp of $G$ from within a fundamental region $\mathscr{R}$. Then $f$ has a Fourier expansion of the form

$$
f(z)=\sum_{n+k>0} c(n) e((n+k) z / \lambda) \quad \text { where } e(z)=e^{2 \pi i z}
$$

and $k$ is defined by

$$
\nu\left(S^{\lambda}\right)=e(k), \quad 0 \leq k<1,
$$

with $S^{\lambda} z=z+\lambda$ being the minimal positive translation of $G$.

The Bers spaces $A_{r}^{p}(G)$ of $p$-integrable and bounded forms are defined as follows. A function $f$ belongs to $A_{r}^{p}(G)$ if $f$ is analytic in $\mathscr{H}$, satisfies (1), and

or

$$
\|f\|_{p}^{p}=\frac{1}{|G(1): G|} \iint_{\mathscr{R}} y^{p r / 2-2}|f(z)|^{p} d x d y<\infty, \quad p<\infty
$$

$$
\|f\|_{\infty}=\sup _{z \in \mathscr{H}} y^{r / 2}|f(z)|<\infty .
$$

Received by the editors March 30, 1986 and, in revised form, March 28, 1987.

1980 Mathematics Subject Classification (1985 Revision). Primary 11F11; Secondary 11 F30. 
The existence of the above norms forces the elements of $A_{r}^{p}(G)$ to be cusp forms. When $p=\infty, A_{r}^{\infty}(G)$ is the space of bounded forms. When $p=1$, one writes $A_{r}(G)$ for $A_{r}^{1}(G)$.

2. Lower bounds. We begin by deriving lower bounds for $\|f\|_{1},\|f\|_{2}$, and $\|f\|_{\infty}$ in terms of the Fourier coefficients of the cusp form.

THEOREM 1. Let $f_{r}$ be a cusp form on $G$ of weight $r$ with Fourier coefficients $c(n)$. Then

$$
\begin{gathered}
\left\|f_{r}\right\|_{1} \geq|c(n)| \lambda^{r / 2} \frac{\Gamma(r / 2-1,2 \pi(n+k) / \lambda)}{|G(1): G|(2 \pi(n+k))^{r / 2-1}} \quad n+k>0, \\
\left\|f_{r}\right\|_{2}^{2} \geq\left|\sum_{s+t=n} c(s) c(t)\right| \lambda^{r} \frac{\Gamma(r-1,2 \pi(n+2 k) / \lambda)}{|G(1): G|(2 \pi(n+2 k))^{r-1}} \quad n+k>0 .
\end{gathered}
$$

In particular, if $k=0, c(1) \neq 0$ and $G=G(1)$, then

$$
\left\|f_{r}\right\|_{2}^{2} \geq|c(1)|^{2} \frac{\Gamma(r-1,4 \pi)}{(4 \pi)^{r-1}} .
$$

Finally,

$$
\left\|f_{r}\right\|_{\infty} \geq|c(n)|(\lambda r /(4 \pi(n+k) e))^{r / 2} \quad n+k>0 .
$$

Here $\Gamma(a+1, x)=\int_{x}^{\infty} y^{a} e^{-y} d y$ is an incomplete gamma function.

PROOF. To prove (2) we first note that

$$
c(n) e^{-2 \pi(n+k) y / \lambda}=\lambda^{-1} \int_{0}^{\lambda} f_{r}(z) e^{-2 \pi i(n+k) x / \lambda} d x
$$

from which it follows that

$$
\begin{aligned}
\lambda^{r / 2}|c(n)| \frac{\Gamma(r / 2-1,2 \pi(n+k) / \lambda)}{(2 \pi(n+k))^{r / 2-1}} & =\lambda|c(n)| \int_{1}^{\infty} y^{r / 2-2} e^{-2 \pi(n+k) y / \lambda} d y \\
& \leq \int_{1}^{\infty} \int_{0}^{\lambda} r^{r / 2-2}\left|f_{r}(z)\right| d x d y \\
& \leq\left\|f_{r}\right\|_{1}|G(1): G| .
\end{aligned}
$$

A similar argument yields (3); and (5) is merely Hecke's classical coefficient estimate, $c(n)=O\left(n^{r / 2}\right)$, with the constant made explicit.

REMARK. When $r=12$ and $\nu=1$, the only cusp form for the full modular group is the classical discriminant function $\Delta(z)$. In [2] it is shown that

$$
\|\Delta\|_{1}=.00070225689, \quad\|\Delta\|_{\infty}=2.02716776 \times 10^{-3} .
$$

Zagier [6] calculates that

$$
\|\Delta\|_{2}^{2}=1.03536205679 \times 10^{-6} .
$$

From (2) and (5), both with $n=1$, and (4) we have

$$
\begin{gathered}
\|\Delta\|_{1} \geq .0006101, \quad\|\Delta\|_{\infty} \geq 1.879583 \times 10^{-3}, \\
\|\Delta\|_{2}^{2} \geq .855 \times 10^{-6} .
\end{gathered}
$$

Hence, the estimates in Theorem 1 do yield fairly reasonable numerical results given that the value of only the first nonzero Fourier coefficient is used. 
3. Upper bounds. In order to obtain upper bounds on the various norms of cusp forms it is reasonable to make use of upper bounds on the Fourier coefficients. However, most of the classical Fourier coefficient estimates are inapplicable because of their dependence on a norm of $f_{r}$. For instance, Rankin's [4, p. 129] famous estimate $c(n)=O\left(n^{r / 2-1 / 5}\right)$, valid for any weight and multiplier system as long as $G$ is a congruence subgroup, depends on $\left\|f_{r}\right\|_{2}$. More recently, Iwaniec [1] showed that $c(n)=O\left(n^{r / 2-2 / 7} d(n) \log ^{2}(2 n)\right)$ for $n$ square-free, weights which are half of an odd integer, and groups $G=\Gamma_{0}(N), N \equiv 0(\bmod 4)$. However, he assumes that $f_{r}$ is normalized so that $\left\|f_{r}\right\|_{2}=1$.

Alternatively, estimates of the form $c(n)=O\left(n^{r / 2-1 / 4+\varepsilon}\right)$ are available (see [3], for instance) when $r=k / 2, k$ an integer, the multiplier system is of arithmetic type, and $G$ is a congruence subgroup. However, the implied constant does depend on the weight $r$ whether the estimate is obtained using Poincare series or using the Hardy-Littlewood circle method. In both cases, the resulting norm estimates are not nearly as good as those in Theorems 2 and 3.

As a result, it appears best to restrict to those cusp forms on the full modular group whose coefficients satisfy the Ramanujan-Petersson coefficient estimate as proved by Deligne; namely,

$$
|c(n)| \leq d(n) n^{(r-1) / 2}
$$

where $d(n)$ is the divisor function. In particular, $r$ is an even integer, the multiplier system is identically one, $f_{r}$ is an eigenform of the Hecke operators, and $c(1)$ is normalized so that $c(1)=1$.

THEOREM 2. Let $f_{r}$ be a cusp form on the full modular group of even integral weight $r$ with multiplier system $\nu=1$. If the Fourier coefficients of $f_{r}$ satisfy (6), then

$$
\left\|f_{r}\right\|_{\infty} \leq(r / 4 \pi e)^{r / 2}(2 r / \pi \sqrt{3})^{1 / 2}(\log r+A)
$$

where $A$ is a constant dependent of $r$.

ProOF. We first note that

$$
\begin{aligned}
y^{r / 2}\left|f_{r}(z)\right| & \leq y^{r / 2} \sum_{n=1}^{\infty}|c(n)| e^{-2 \pi n y} \\
& \leq(r / 4 \pi e)^{r / 2} \sum_{n<r / 2 \pi \sqrt{3}} \frac{|c(n)|}{n^{r / 2}}+(\sqrt{3} / 2)^{r / 2} \sum_{n>r / 2 \pi \sqrt{3}}|c(n)| e^{-\pi \sqrt{3} n}
\end{aligned}
$$

since $g(y)=y^{r / 2} e^{-2 \pi n y}$ attains its maximum at $y=r /(4 \pi n)$. Then, by (6),

$$
\begin{aligned}
\left\|f_{r}\right\|_{\infty} & \leq(r / 4 \pi e)^{r / 2} \sum_{n<r / 2 \pi \sqrt{3}} \frac{d(n)}{n^{1 / 2}}+(\sqrt{3} / 2)^{r / 2} \sum_{n>r / 2 \pi \sqrt{3}} d(n) n^{(r-1) / 2} e^{-\pi \sqrt{3} n} \\
& =S_{1}+S_{2} .
\end{aligned}
$$

Since $\sum_{n \leq x} d(n)=x \log x+O(x)$, by Abel summation

$$
\begin{aligned}
S_{1} & =(r / 4 \pi e)^{r / 2}\left\{2(r / 2 \pi \sqrt{3})^{1 / 2} \log (r / 2 \pi \sqrt{3})+O\left(r^{1 / 2}\right)\right\} \\
& =(r / 4 \pi e)^{r / 2}(2 r / \pi \sqrt{3})^{1 / 2}(\log r+O(1)) .
\end{aligned}
$$


To estimate $S_{2}$, note that since $d(n)=O\left(n^{1 / 2}\right)$,

$$
\begin{aligned}
S_{2} & =O\left((\sqrt{3} / 2)^{r / 2} \sum_{n>r / 2 \pi \sqrt{3}} n^{r / 2} e^{-\pi \sqrt{3} n}\right) \\
& =O\left((r / 4 \pi e)^{r / 2}+(\sqrt{3} / 2)^{r / 2} \int_{r / 2 \pi \sqrt{3}}^{\infty} t^{r / 2} e^{-\pi \sqrt{3} t} d t\right) \\
& =O\left((r / 4 \pi e)^{r / 2}+(r / 2) ! /(2 \pi)^{r / 2}\right) \\
& =O\left((r / 4 \pi e)^{r / 2} r^{1 / 2}\right) .
\end{aligned}
$$

Combining the estimates for $S_{1}$ and $S_{2}$ gives the upper bound on $\left\|f_{r}\right\|_{\infty}$ and also shows that $S_{1}$ is the dominant term, as one would expect.

THEOREM 3. Suppose $f_{r}$ is a modular cusp form of even integral weight $r$ with $\nu=1$ whose coefficients satisfy (6). For any $\varepsilon>0$, let $C_{\varepsilon}$ be the constant occurring in the estimate $d(n) \leq C_{\varepsilon} n^{\varepsilon}$. Then,

$$
\begin{aligned}
\left\|f_{r}\right\|_{1} \leq & \left(C_{\varepsilon} /(2 \pi)^{r / 2-1}\right) \\
& \times\left(\frac{\Gamma((r+1) / 2+\varepsilon, \pi \sqrt{3})}{(3 / 2+\varepsilon)(\pi \sqrt{3})^{3 / 2+\varepsilon}}+\frac{\Gamma((r-1) / 2+\varepsilon, \pi \sqrt{3})}{(\pi \sqrt{3})^{1 / 2+\varepsilon}}\right)
\end{aligned}
$$

and

$$
\begin{aligned}
\left\|f_{q}\right\|_{2}^{2} \leq & \left(\left(C_{\varepsilon}\right)^{2} /(4 \pi)^{r-1}\right) \\
& \times\left(\frac{\Gamma(r+2 \varepsilon, 2 \pi \sqrt{3})}{(2 \pi \sqrt{3})^{1+2 \varepsilon}(1+2 \varepsilon)}+\frac{\Gamma(r+2 \varepsilon+1,2 \pi \sqrt{3})}{(2 \pi \sqrt{3})^{2 \varepsilon}}\right) .
\end{aligned}
$$

PROOF. We first note that

$$
\begin{aligned}
\left\|f_{r}\right\|_{1} & =\iint_{\mathscr{R}} y^{r / 2-2}|f(z)| d x d y \\
& \leq \int_{\sqrt{3} / 2}^{\infty} y^{r / 2-2} \sum_{n=1}^{\infty}|c(n)| e^{-2 \pi n y} d y \\
& \leq C_{\varepsilon} \sum_{n=1}^{\infty} n^{(r-1) / 2+\varepsilon} \int_{\sqrt{3} / 2}^{\infty} y^{r / 2-2} e^{-2 \pi n y} d y
\end{aligned}
$$

by (6)

$$
=\left(C_{\varepsilon} /(2 \pi)^{r / 2-1}\right) \sum_{n=1}^{\infty} n^{1 / 2+\varepsilon} \Gamma(r / 2-1, \pi n \sqrt{3}) .
$$

Then, since

$$
\sum_{n \leq x} n^{1 / 2+\varepsilon}<x^{3 / 2 \varepsilon} /(3 / 2+\varepsilon)+x^{1 / 2+\varepsilon}
$$

a summation by parts shows that

$$
\begin{aligned}
& \sum_{n=1}^{\infty} n^{1 / 2+\varepsilon} \Gamma(r / 2-1, \pi n \sqrt{3}) \\
& \quad<\frac{\Gamma((r+1) / 2+\varepsilon, \pi \sqrt{3})}{(3 / 2+\varepsilon)(\pi \sqrt{3})^{3 / 2+\varepsilon}}+\frac{\Gamma((r-1) / 2+\varepsilon, \pi \sqrt{3})}{(\pi \sqrt{3})^{1 / 2+\varepsilon}} .
\end{aligned}
$$

(7) follows immediately; and (8) is proved similarly. 
4. Conclusion. By combining Theorems 1, 2, and 3, one obtains upper and lower bounds on the various norms of cusp forms on the full modular group whose Fourier coefficients satisfy (6). It is worth pointing out that there is room for improvement in these bounds. For instance, for $\left\|f_{r}\right\|_{2}^{2}$ the lower bound is $\Gamma(r-1,4 \pi) /(4 \pi)^{r-1}$ whereas the upper bound is $O\left(r^{1+2 \varepsilon} \Gamma(r-1,4 \pi) /(4 \pi)^{r-1}\right)$.

The other natural normalization of cusp forms when viewed as integrable and bounded forms is to require that the respective norm be one. In this case, Theorems 1,2 , and 3 (where $c(n) / c(1)$ now satisfies (6)) can be reinterpreted as giving information on the size of $|c(1)|$. In particular, if $\left\|f_{r}\right\|_{2}=1$, then

$$
\begin{aligned}
|c(1)|^{2} & \leq(4 \pi)^{r-1} / \Gamma(r-1,4 \pi) \\
& \leq(4 \pi)^{r-1} e^{4 \pi} /(4 \pi)^{r-2}=4 \pi e^{-4 \pi} .
\end{aligned}
$$

Again, the question of the exact order of growth of $|c(1)|$ as a function of $r$ remains open.

\section{REFERENCES}

1. H. Iwaniec, Fourier coefficients of modular forms of half-integral weight, Invent. Math. 87 (1987), 385-401.

2. L. A. Parson, A lower bound for the norm of the theta operator, Math. Comp. 41 (1983), 683-685.

3. Math. Soc. 217 (1976), 329-350.

4. R. A. Rankin, Modular forms and functions, Cambridge Univ. Press, Cambridge, 1977.

5. R. A. Smith, The $L^{2}$-norm of Maass wave functions, Proc. Amer. Math. Soc. 82 (1981), 179-182.

6. D. B. Zagier, Modular forms whose Fourier coefficients involve zeta-functions of quadratic fields, Modular Functions of One Variable VI (J.-P. Serre and D. B. Zagier eds.), Springer Lecture Notes, no. 627, 1977, 105-169.

Department of Mathematics, Ohio State University, Columbus, Ohio 43210 